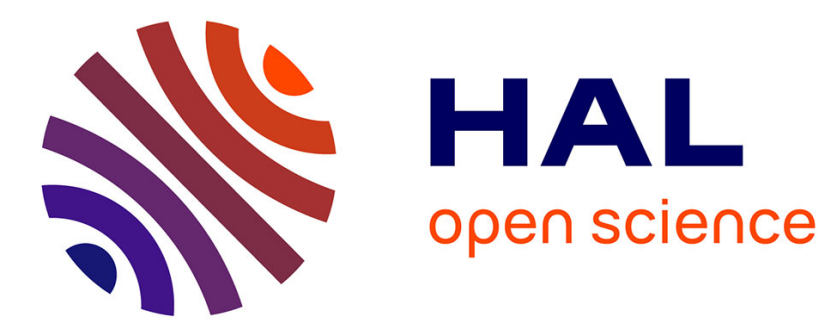

\title{
Three-stage multiscale modelling of the NMDA neuroreceptor
}

Francesco Di Palma, Sauro Succi, Fabio Sterpone, Marco Lauricella, Franck Pérot, Simone Melchionna

\section{> To cite this version:}

Francesco Di Palma, Sauro Succi, Fabio Sterpone, Marco Lauricella, Franck Pérot, et al.. Three-stage multiscale modelling of the NMDA neuroreceptor. Molecular Physics, In press, 10.1080/00268976.2021.1928312 . hal-03364615

\section{HAL Id: hal-03364615 https://hal.science/hal-03364615}

Submitted on 13 Oct 2021

HAL is a multi-disciplinary open access archive for the deposit and dissemination of scientific research documents, whether they are published or not. The documents may come from teaching and research institutions in France or abroad, or from public or private research centers.
L'archive ouverte pluridisciplinaire HAL, est destinée au dépôt et à la diffusion de documents scientifiques de niveau recherche, publiés ou non, émanant des établissements d'enseignement et de recherche français ou étrangers, des laboratoires publics ou privés. 


\title{
Three-stage multiscale modelling of the NMDA neuroreceptor
}

\author{
Francesco Di Palma ${ }^{1}$, Sauro Succi ${ }^{1}$, Fabio Sterpone ${ }^{2}$, \\ Marco Lauricella ${ }^{3}$, Franck Pérot ${ }^{4,6}$, Simone Melchionna ${ }^{4,5}$ \\ ${ }^{1}$ Istituto Italiano di Tecnologia, Central Research Labs, \\ Via Morego 30, 16163 Genova, Italy \\ ${ }^{2}$ Laboratoire de Biochimie Théorique, \\ UPR 9080, CNRS Université Denis Diderot, \\ Sorbonne Paris Cité, IBPC, Paris, France \\ ${ }^{3}$ Istituto Applicazioni Calcolo M. Picone CNR, \\ Via dei Taurini 19, 00185 Rome, Italy \\ ${ }^{4}$ Istituto Sistemi Complessi CNR, Via dei Taurini 19, 00185 Rome, Italy \\ ${ }^{5}$ MedLea Srls, Italy and \\ ${ }^{6}$ Lexma Technology LLC, USA
}

\begin{abstract}
We present a new multistage method to study the N-Methyl-D-Aspartate (NMDA) neuroreceptor starting from the reconstruction of its crystallographic structure. Thanks to the combination of Homology Modelling, Molecular Dynamics and Lattice Boltzmann simulations, we analyse the allosteric transition of NDMA upon ligand binding and compute the receptor response to ionic passage across the membrane.
\end{abstract}




\section{INTRODUCTION}

Modern biological sciences are in a relentless search to unveil the molecular mechanisms of biological activity, their dis-functionalities and the ensuing diseases, so as to propose and develop possible remedies. Computer models have a crucial say in this endeavour, as they can often access regions of parameter space which are beyond reach of direct experimentation. In particular, molecular dynamics simulations have a long and time-honoured history in the study of biomolecular assemblies culminating with the 2013 Nobel Prize in Chemistry Karplus et al. [1].

The field shows no sign of flagging; quite on the contrary it is witnessing burgeoning progress in multiple directions, particularly in terms of capabilities to tackle larger systems over longer temporal scales. Remarkable examples in point are the recent simulations of biological systems is the 100-million atom-scale model of an entire cell organelle, a photosynthetic chromatophore vesicle from a purple bacterium Rochaix [2] or the study of the N-Methyl-D-Aspartate (NMDA) neuroreceptor by the DE Shaw research group Song et al. [3]. These outstanding achievements were obtained by leveraging the method of Molecular Dynamics (MD), whereby all atoms in the simulation are taken into account, with a massive computational effort, often undertaken on special hardware.

Such achievements unquestionably push the frontiers of simulation further ahead and pave the way to disruptive progress in many areas of biology and medicine. In particular, the possibility of simulating large systems is key to neuroscience, where the response of neuroreceptors to binding with natural or synthetic ligands can reveal the allosteric transitions in the membrane protein and how the channel pore function, with the modulation of the ionic passage. NMDA receptors, in particular, play a critical role in brain development and function, including learning and memory formation Trimble [4], Burnell et al. [5]. Dysfunctional NMDA is involved in various neurological disorders, such as Alzheimer's disease, depression, stroke, epilepsy and schizophrenia. Therefore, the computational capability to tackle large-scale protein transitions and ion passage, has major potential to study many neurological diseases and design new drugs through medicinal chemistry methods. Some initial studies have indeed tackled the study of NMDA by a brute force MD simulations that, while showing some degree of success, have also highlighted the difficulty of covering the complete times scale of the allosteric and functional response Song et al. [3], Dai and 
Zhou [6], Zheng et al. [7], Palmai et al. [8].

Under physiological conditions, the opening of the receptor ion channel involves the concurrent binding of glycine and glutamate and relief of magnesium block at the ion channel pore by membrane depolarisation. The resulting calcium flux triggers a cascade of signal transduction that is necessary for synaptic plasticity.

Multiscale methods offer a strategic advantage in simulating such receptors that lay at the basis of many basic neurological phenomena. The reasons is that neuroreceptors are large molecular assemblies with large stretches of membranes separating intra and extracellular regions and receptors (proteins) as gates. Binding small molecules (neurotransmitters) triggers spatially small but functionally very important structural changes (allosteric transitions) that open/close the gates to the passage of ions.

From the neurological viewpoint, it is key to quantify the allosteric response to the binding of nanomolar quantities of neurotransmitters. These can be natural or artificial, with important applications to medicinal chemistry. Once the allostery is measured and quantified, one can then measure passage of ions in the receptor pore. To this purpose, electrokinetics raises a significant challenge, as it implies the presence of blocking elements (Magnesium for NMDA), since the process is an activated one. To accomplish such an ambitious program, all-atoms simulations fall short of covering the wide stretch of spatial and temporal scales involved, ranging from picoseconds to minutes and with a number of atomic degrees of freedom in excess of millions. In essence, reproducing binding affinity (thermodynamics), allosteric transitions (large-scale, fine and coarse atomic motion) and electro-kinetics, is a task that involves too widely disparate scales to be amenable to a single level of representation and simulation.

For these reasons, we have devised a three-stage multiscale pipeline to study neuroreceptors, starting from the reconstruction of the protein structure, as obtained from incomplete crystallographic data, to analyse the binding affinity of NMDA to its ligands, followed by the quantification of the allosteric motion, and finally to assess the modulation of the ionic passage.

Our approach begins by using homology modelling techniques to reconstruct the NMDA configuration in the phospholipidic environment, the simulation of the allosteric events by means of MD and finally by the study of the electrokinetic ion transport by means of a Lattice Boltzmann approach. The main novelty of our approach relies on the use of LB 
electrokinetics to analyse the flow of ions in a probabilistic way. While other strategies can be powerful to study the passage of single or multiple charges in ion channels via the potential of mean force method Roux et al. [9] or in a statistical sense, by solving the Poisson-Nernst-Planck equation Kurnikova et al. [10], our approach takes into account the complete hydrodynamic modes of the saline solution flowing through the receptor matrix. This permits a direct assessment of the effect of electrostatic forces and convection on the three-component solution made of neutral, cationic and anionic species. The approach thus allows selecting a large set of protein configurations so that the prediction of flux based on configuration allows direct comparative analysis with purely steric approaches Smart et al. [11]. Finally, the proposed method can be enriched by combining the sampling of configurational space with adequate simulations of all atoms or coarse grained models, in order to follow the flow in different conditions.

The present three-stage approach is entirely novel in concept and application and demonstrates, for the first time to the best of our knowledge, the possibility to tackle complex biological processes occurring in neuroscience can be tackled, thus providing a whole wealth of microscopic, dynamic and functional information, otherwise unaccessible to current experimental techniques.

\section{COMPUTATIONAL APPROACH}

As shown in Fig 1, our computational approach involves a highly orchestrated network of computational methods and software tools, which can broadly classified within three basic steps:

1. Structure modelling (Homology Methods);

2. Mechanical stability (Molecular Dynamics and Molecular Mechanics);

3. Ionic transport (Lattice Boltzmann).

The whole three-stage procedure is genuinely multiscale, as it spans about six decades in time, from nanoseconds up to milliseconds, thus reaching up to timescales of physiological relevance. The ultimate outcome of clinical relevance is the dose response relationship, namely the current flowing across the neurotransmitter as a function of the dose of ligand. 
To complete the three stages of the flowchart, we used three different software packages: Modeller Eswar et al. [12] for Homology Modeling and Gromacs Abraham et al. [13] for All-Atoms MD. Most of simulations are based on the software Moebius from Lexma Technology, as it allows multiphysics simulations of multicomponent fluids and particles. Therefore, Moebius was then used for the Coarse-Grained MD and for the Lattice Boltzmann simulations. We utilised both the CPU and GPU architectures to accomplish the computing-intensive runs, as available on both the Gromacs and Moebius softwares.

\section{A. Structure Modelling}

The first stage of the flowchart pertains to characterising the molecular structure of the neuroreceptor, consisting of the protein embedded in the cellular membrane. Homology modelling allows to construct the atomic-resolution model of NMDA GluN1A/GluN2B starting from its amino acid sequence and the available crystallographic three-dimensional structure (PDB id: 4PE5) Karakas and Furukawa [14]. For crystallographic data from membrane proteins with a large amount of missing structures, such as for NMDA, poses a major challenge to homology modelling.

Reconstructing the missing loops is accomplished by the high precision DOPE-HR modelling protocol Shen and Sali [15], whereby the atomic-resolution model of the target protein from its amino acid sequence is constructed on the basis of the experimental threedimensional structure of a template homologous protein at $3.96 \AA$ resolution (PDB id: 4PE5 in this specific case) Karakas and Furukawa [14] The reconstruction relies on the identification of known portions of the structure from homologous receptors, acting as templates that resemble the structure of the query sequence and on an alignment that maps residues to residues in the template sequence. The identification of one or more known structures resembling the one of the query sequence and the alignment of residues in the query sequence to residues in the template sequence, benefits from the fact that NMDA structure is conserved amongst homologues as well as on the loop reconstruction algorithm (as quantified by DOPE-HR). Such a method provides the slowest but most accurate available refinement method to obtain better quality loops (i.e. by selecting the refine.slow large or refine.very slow options depending on the length of the missing loops). 


\section{B. Mechanical Stability and Allostery}

The second stage of the flowchart consists of assessing the stability of the aforementioned molecular complex, including its interactions with the water solvent, and assessing the allosteric transition. Once ligands bind to the specific receptor sites, NMDA changes its shape, and altering the affinity for a ligand at a second site (e.g., either a receptor or a binding site); the ability of an effector molecule (ligand) to change the conformation and activity of a protein.

In allostery, the function of a receptor is modified by the interaction with its ligands, not only at the active site but also at a spatially distinct site of different specificity. In allostery, the interaction of the functional sites results in an altered affinity of ligand binding, thus depending on the dynamic interaction with the substrate. Ideally the conformational changes induced by the binding of the allosteric effector can be finely followed by brute force MD simulations at atomistic resolution. For instance, if the allosteric response path is of interest, starting from the substrate free equilibrated structure it is possible to introduce the effector and follow in time its conformational changes and fluctuations. This is the strategy used in our approach. In other situations, where both the APO and HOLO states are available, the thermal fluctuations in each state, and possible state interconversion can be simulated with brute force MD or by enhanced sampling techniques (e.g. parallel tempering, thermodynamic integration, metadynamics, etc.).

In our approach based on the all atoms mode, we simulated both the APO (ligand free) and the HOLO (ligand bound) versions of NMDA. For the HOLO version, we positioned two Glutamate (GLU) and two Glycine (GLY) neurotransmitters molecules in the NMDA pockets of the relaxed model. This was obtained by docking them by means of the Chimera software chain-by-chain superimposition to achieve suboptimal overlap of the protein domains.

Insertion of the in vacuum minimised NMDA model in a lipid bilayer in order to create the protein/membrane system was done by using the Charmm-Gui membrane builder generator Jo et al. [16], Wu et al. [17]. Embedding in the phospholipid membrane was done by accurately determining at first the NMDA transmembrane portion on Orientation of Proteins in Membranes database (http://opm.phar.umich.edu/server) Lomize et al. [18] in order to take advantage of this selection during the Charmm-Gui procedure. The membrane of 
homogenous phospholipids (i.e. POPC) was constructed along the xy plane $(\mathrm{x}=\mathrm{y}=14 \mathrm{~nm})$ placing NMDA major axis along the $\mathrm{z}$ direction $(20.5 \mathrm{~nm}$ with a water buffer layer of 1.75 $\mathrm{nm}$ on top and bottom of the system) of the rectangular box at the proper z-height to cover the membrane-spanning segments of the protein according to OPM result. The final system is composed of 376782 atoms: 50574 for NMDA, 506 POPC lipids, 85962 TIP3P molecules, 286 potassium ions and 232 chlorine ions, thus resulting in a $0.15 \mathrm{M}$ ionic concentration. Using the Charmm-Gui suggested standard protocol Lee et al. [19], the system has been minimised (5000 steep descent steps) and equilibrated with Gromacs 2018 Abraham et al. [13] and CHARMM36m force field Huang and MacKerell Jr [20] with the following protocol: five MD simulations of variable length (25000, 25000, 25000, 50000 and 50000 steps) with lipid position restraints of decreasing strength (1000 to $200 \mathrm{~kJ} \mathrm{~mol}^{-1} \mathrm{~nm}^{-2}$ ) and similarly for dihedral (400 to $100 \mathrm{~kJ} \mathrm{~mol}^{-1} \mathrm{rad}^{-2}$ ) and protein backbone restraints (2000 to $200 \mathrm{~kJ}$ $\mathrm{mol}^{-1} \mathrm{~nm}^{-2}$ ). The first two simulations were done at constant temperature and volume, the remaining three simulations at constant temperature and pressure. A sixth MD simulation of 50000 steps for equilibration was performed without restraints.

However, the high resolution of the full atomistic approach is often limiting for very large systems. It is convenient therefore to use a simplified coarse-grained model. This is particularly challenging for membrane proteins that, for a proper treatment of allosteric process, an adequate level of controlled flexibility of the protein scaffold is required. In our approach we tested the capability of the OPEP CG force field Chebaro et al. [21], Sterpone et al. $[22,23]$ to reproduce the main fluctuations and conformational changes associated to the allosteric transition by comparing the results with atomistic modelling.

The OPEP force field has been designed to model peptide and protein folding and aggregation in solution. It is a multi-scale model that reserves an atomistic description to the backbone atoms and reduces the side chain amino acid to a unique bead. OPEP was designed, and progressively improved, for modelling soluble proteins Chebaro et al. [21], Sterpone et al. [22, 23] but its coupling with an explicit membrane environment is under way. For the purpose of the present application this is not a limitation. In fact, the conformational changes induced by the allosteric effector are localised in the extra-membrane part of the protein, and in order to assure the stability of the trans-membrane region several constrained were applied so to mimic the spatial embedding of the membrane without its 
explicit representation.

The test was performed on two models. In the first one the protein motion is fully flexible and its motion is controlled by the OPEP hamiltonian. The flexibility is key for monitoring the loops motions in the extra-membrane regions that control the allosteric response. The second model uses a more radical simplification, and is based on a floppy elastic network of the protein. On top of the elastic network, non-bonded sites interact via the OPEP non-bonded potential. To be noted that in several previous works the characterisation of protein allosteric paths are modelled by considering elastic networks Rocks et al. [24]. In the CG approach for the allosteric effectors (GLN and GLY amino acids) instead we used the same OPEP model but their location in the binding site was restrained. It is worth noting that the OPEP force field was already successfully coupled to the hydrodynamic description based on LB Sterpone et al. [25], allowing the investigation of complex processes like amyloid aggregation Chiricotto et al. [26], Chiriccotto et al. [27], protein crowding Sterpone et al. [22], Timr et al. [28] and unfolding under shear flow Sterpone et al. [29], Languin-Cattoen et al. [30].

Finally, and relevant, for the present application where different resolutions are compared, it is worth mentioning that the behaviour of the OPEP force field has been already contrasted to all atoms simulations in several contexts, from protein folding and aggregation, to thermal and mechanical unfolding Sterpone et al. [22, 23], Languin-Cattoen et al. [30].

\section{Ionic Currents}

Finally, the third stage pertains to the simulation of the ionic currents within the neuroreceptor, which is in charge of computing the conductivity of the neuroreceptor as a function of the applied voltage or salinity and for varying configurations of the receptor.

The Poisson-Nernst-Planck (PNP) equation describes the drift-diffusion of ions in liquid solutions under the influence of an electric driving field. The method can handle channel inhomogeneities such as narrowings and/or non uniformities in the chemical nature of the confining surface Kurnikova et al. [10]. The PNP model uses two diffusion equations for the species densities and the self-consistent electric potential, being the solution of a Poisson problem. The PNP equation is highly non local and requires a numerical solution. At its core, the approach neglects advection of the solution and the resulting electro-osmotic effects, 
whose intensity is unknown a priori in highly confined electrobiochemical processes. While the effects of hydrodynamic modes in saline solution effect in ion channels cannot be ruled out from the start for a given neuroreceptor under study, it is safe to avoid using uncontrolled approximations in the numerical method. For isntance, the effect of hydrodynamics on highly confined electrokinetic flows has been observed in experiments and confirmed by simulations Fyta et al. [31]. The Lattice Boltzmann (LB) method provides a particularly useful approach to investigate the ionic response since it is rooted in the mesoscopic description of matter which perfectly maps the current representation of the receptor and its environment Succi $[32]$.

LB is a very convenient computational approach, since it is based on a over a Cartesian grid, namely a uniform mesh based on cubic voxels. It is highly adaptable to reproduce the flow structure of single and multi-component system under the action of external or internal forces. In particular, LB has been shown in the past to be capable of reproducing the electro-kinetics of saline solutions in model devices with non-trivial geometries and locally charged surfaces Marconi and Melchionna [33], Melchionna and Marconi [34].

While the all-atom and potential of mean force approaches automatically include all electrostatics from atomistic pairwise interactions, when utilising a field-based description numerical accuracy should be treated with care. The reason stems from the fact that the electrostatic Poisson equation is defined in the biomolecules and in the solvent, while the fluid flow equation are defined only in the solvent. On the other hand, if the fluid equations are solved in the entire simulation domains singularities arise from the source charges inside biomolecules. A natural approach is to remove the singular component of the electrostatic potential induced by the permanent charges, so that well-posed numerical equations are handled. Recently, a method was devised for the time integration for the unsteady PNP equations that alleviates the problem stemming from the stiff matrices in the finite element approximations. The results are both accurate and stable Lu et al. [35]. A different approach applies to the LB scheme. Here, at variance with the PNP method, electrostatic forces act directly on the solvent via a direct forcing. Therefore, electrostatic accuracy depends on the mesh resolution that can be shown to ameliorate with the mesh spacing as $\sim \Delta x^{2}$. Electrokinetic simulations on synthetic nanopores and on silica channels have been shown that the spacing usually employed in LB simulations are sufficient to provide highly accurate results under various conditions of electro-osmotic flows Marconi 
and Melchionna [33], Melchionna and Succi [36], Marini Bettolo Marconi and Melchionna [37]. Therefore, we compared the characteristic curve obtained with the LB method to experimental data Cui et al. [38], as reported in Fig. 6, showing a close quantitative match for such a complex biomolecular system.

The case of the NMDA receptor, however, presents a fairly non trivial geometry within the pore region and highly localised forces $F(x)$ at position $x$ stemming from charged or neutral atomic groups. In addition, the local electrostatic forces can be locally intense, often exhibiting rapid spatial modulations of the electrolytic densities due to the formation of disordered double layers. Such scenario poses several challenges to the computational scheme, particularly by endangering numerical stability as dictated by the Courant-Friedrich-Lewy stability condition $F(x) \lesssim m \Delta x / \Delta t^{2}$. It is well-known that the presence of unit charges in simulation cannot be simulated by a direct LB approach Raafatnia et al. [39] without proper treatment to enhance its stability.

To circumvent such limitations, we employed a multi-component Entropic Lattice Boltzmann Method, a powerful variant of the basic Lattice Boltzmann method based on a selfconsistent tuning of the relaxation parameter so as to ensure compliance with local entropy growth (H-theorem).

The LB approach to simulating the saline solution is based on tracking the evolution of each fluid component, with the index $\alpha=0,1,2$ labelling the neutral aqueous medium (0), and the positively (1) and negatively charged components (2), via the discretised form of the density distribution function, named populations $f_{p}^{\alpha}(x, t)$. Here, $t$ denotes the temporal coordinate and subscript $p$ labels a set of discrete speeds $c_{p}$ connecting the mesh points to its neighbours. The two ionic components are monovalent and characterised by charges density $n^{\alpha}$ and velocity $u^{\alpha}$ and, given the barycentric velocity $u=\frac{\sum_{\alpha} n^{\alpha} u^{\alpha}}{\sum_{\alpha} n^{\alpha}}$, the relative velocity being denoted $\delta u^{\alpha}=\left(u^{\alpha}-u\right)$. The neuroreceptor and the membrane are described by the collection of particles at position $\vec{r}_{i}$ being neutral or partially charged with charge $q_{n}$ valence $z_{n}$ provided by the force field.

In standard multicomponent LB the dynamics of each component then follows its own evolution equation:

$$
f_{p}^{\alpha}\left(r+c_{p}, t+1\right)=f_{p}^{\alpha}(r, t)+\omega\left(f_{p}^{\alpha, e q}-f_{p}^{\alpha}\right)+S_{p}^{\alpha}
$$


where $\omega$ is a relaxation frequency related to the kinematic viscosity as $\nu=c^{2}\left(\frac{1}{\omega}-\frac{1}{2}\right)$, $c=1 / \sqrt{3}$ the lattice speed of sound, and the Maxwellian equilibrium given by Marconi and Melchionna [33, 40],

$$
f_{p}^{\alpha, e q}=w_{p} n^{\alpha}\left[1+\frac{\delta u^{\alpha} \cdot c_{p}}{c^{2}}+\frac{\left.\left(\delta u^{\alpha} \cdot c_{p}\right)^{2}-c^{2}\left(\delta u^{\alpha}\right)^{2}\right)}{2 c^{4}}\right]
$$

and $w_{p}$ is a set of normalised weights. The force term is $S_{p}^{\alpha}=$ $w_{p} n^{\alpha}\left[\frac{F^{\alpha} \cdot c_{p}}{c^{2}}+\frac{\left.\left(c_{p} \cdot u\right)\left(c_{p} \cdot F^{\alpha}\right)-c^{2} F^{\alpha} \cdot u\right)}{c^{4}}\right]$ and the local force $F^{\alpha}=-e z^{\alpha} \nabla \psi-\omega_{d r a g}^{\alpha} \sum_{\beta} \frac{n^{\beta}\left(u^{\alpha}-u^{\beta}\right)}{n}+$ $\gamma u^{\alpha} \tilde{\delta}\left(r_{n}-x\right)$, being the sum of the self-consistent electrostatic forces, the inter-specie drag force characterised by the cross-diffusion coefficient $D^{\alpha}=c^{2} / \omega_{d r a g}^{\alpha}$, and the frictional force exerted by the receptor and membrane atoms on the fluid species, being proportional to the coefficient $\gamma$. In addition, $\tilde{\delta}(x)$ is a function used to smear the particle charge on the LB grid, as used in Immersed Boundary method Peskin [41].

The electrostatic potential $\psi$ is the solution of the Poisson equation, $\nabla^{2} \psi=-\frac{1}{\epsilon}\left[e n^{+}(x)-\right.$ $\left.e n^{-}(x)+\sum_{n} z_{n} q_{n} \tilde{\delta}\left(r_{n}-x\right)\right]$ in the aqueous medium of dielectric permittivity $\epsilon$, $e$ being the unit electronic charge. In this study the LB solution is obtained on a cartesian grid of spacing $0.01 \mathrm{~nm}$, by employing the D3Q19 set of discrete speeds and associated weights, and by smearing particle charges over $2 \times 2 \times 2$ grid points. We chose a ionic cross-diffusivity of $2 \mathrm{~nm}^{-2} / \mathrm{ns}$ and a frictional coefficient $\gamma=0.5 \mathrm{~ns}^{-1}$.

The complete multicomponent Entropic LB evolution is obtained by solving eq. 1 complemented by the minimisation of the lattice H-function $H^{\alpha}\left[f^{\alpha}\right]=\sum_{p} f_{p}^{\alpha} \log \left(f_{p}^{\alpha} / w_{p}\right)$, which additionally provides a small local adjustment to the relaxation frequency $\omega$ to enforce stability. Under operating conditions we found that applying a filtering approach to populations to remove non-hydrodynamic modes Krämer et al. [42] significantly enhances numerical stability Montessori et al. [43], providing the final conditions to simulate saline solutions in presence of unit charges.

\section{RESULTS}

In the following, we provide a summary of the main results of our analysis. 


\section{A. Homology Modelling}

We first considered the tetrameric NMDA GluN1A/GluN2B structure from Rattus Norvegicus reconstructed by Homology Modelling at atomic-resolution model starting from the three-dimensional crystallographic structure Karakas and Furukawa [14].

The structure lacks several loops in the linker region between the ligand-binding domain and the missing loops were reconstructed chain-by-chain using the high precision DOPE-HR modelling protocol Shen and Sali [15], resulting in the most accurate available refinement method to obtain high quality structural stretches.

Subsequently, the receptor was relaxed using an energy minimisation scheme first in vacuum by means of MD via a steepest descent algorithm to overcome/solve bad contacts and improve the overall quality of the structure quality.

Validation of both post-homology model and equilibrated structures was obtained by using MolProbity Williams et al. [44] that provided sterics and geometrical scores (allatom contacts, Ramachandran plot, rotamer outliers, no structural aberrations, intra e inter-molecular knots) for both the modelled structure and the equilibrated system that we have used to initialize the three different sets of simulations. Once relaxed, the receptor was embedded in a phospholipidic membrane and prepared for simulation by surrounding the entire system in water molecules at ambient temperature $(303.15 \mathrm{~K})$ and as density corresponding to $1000 \mathrm{Kg} / \mathrm{m}^{3}$ (as detailed above in the Methods).

\section{B. Molecular Dynamics and Molecular Mechanics simulations}

Once the system was validated, we performed MD simulations at three increasing levels of coarse graining, namely i) All-atom resolution based on CHARMM36m force field, ii) Coarse-grained MD based on OPEP force field, iii) elastic network representation. We simulated both the APO (ligand free) and the HOLO (ligand bound) versions of NMDA.

The atomistic simulations (stable in terms of RMSD, see Fig.4) successfully model the allosteric effect, since upon the binding of the effector (GLN or GLY) we observe the opening of the channel associated to the specific reorganisation of the extra-membrane region. This opening is observed in the timescale of $300 \mathrm{~ns}$ thus resulting in a quite different conformation when comparing the HOLO and APO final structure (see Fig. 4 insets). 
A first-level coarse-grained model was utilised by employing the OPEP force field Sterpone et al. [45] to represent amino acids fully explicitly for the peptidic backbone, inclusive of hydrogen atoms, while the lateral groups are represented by an effective particle. Such representation is particularly accurate for the backbone as it includes stretching, angular and torsional movements together with non-covalent and hydrogen bonds. The pseudoparticles for the lateral groups take into account the sterical and non-covalent interactions, together with explicit representations of the saline bridges.

The CG simulations were performed starting from the minimised, pressurised and equilibrated all-atom conformation and contrasted against the atomistic one taken as reference. We observed a comparable opening of the channel. The result supports the use of this less time consuming model to generate a valid ensemble of configurations for further electrokinetic analysis, as it is confirmed analysing both the HOLO and APO trajectory RMSD (Fig. 4). A detailed description of the trajectories and the conformational motions associated to the allosteric transition is reserved to a further work. The obtained results represent an extra validation of the OPEP force field and potentially extend its applicability and further tests to allosteric and transmembrane systems.

A further level of coarse graining was employed by using an elastic network representation. Here all intramolecular bonding and non-covalent forces were substituted with harmonic interactions that allow for a certain level of protein deformability. Intermolecular forces are still accounted for by means of the OPEP force field. Also in this latter case both simulations, with and without the ligands, resulted stable on the microsecond timescale.

All three models showed to undergo the allosteric transition once the ligands have been positioned in the corresponding pockets.

The transition took place on the $100 \mathrm{~ns}$ timescale and several attempts were observed before NMDA finally reached its stable configuration, which is supposed to be an open pore configuration (see Fig. 4). Importantly, the consensus of the three models to reproduce the transition lends a significant degree of confidence to the overall homology-based reconstruction and simulation models.

With the three representations of the neuroreceptor in place, we were able not only to generate a large number of configurations with the all-atom method, but also to harvest the relaxed structures by replacing the membrane and receptor by order two different levels of coarse graining, the OPEP and elastic network models. 


\section{Ionic passage}

A purely steric analysis of the pore geometry is initially performed by finding the best route for a sphere with variable radius to squeeze through the channel. The method HOLE allows the analysis of the dimensions of the pore running through a structural model of an ion channel Smart et al. [11] where the algorithm uses a Monte Carlo simulated annealing procedure. The method predicts conductance by using a simple empirically corrected ohmic model. However, ion permeation cannot be simply identified from the physical dimensions of the pore. For example, water within narrow hydrophobic pores can modulate permeation without even requiring steric occlusion of the pathway. Better methods have been proposed to account for hydrophobic gating, such as in the CHAP method Klesse et al. [46].

The effective passage of ions can take place in any of the small apertures and crevices present within the receptor matrix, that can be seen as an effective porous medium. Fig. 3 illustrates the steric passage within NMDA for a given receptor conformation. The available volume is rather funnel shaped and the receptor bottleneck is very narrow, with a lateral aperture being smaller than one Angstrom. By analysing the time evolution of the bottleneck distance, along the all-atom simulations computed as the distance between the two GluN1A subunits considering the CoM of Val632 and Val2244, and between the GluN2B subunits Ile1426 and Ile3039, as reported in Fig. 5, we observe a differentiated behaviour between the APO and HOLO states, suggesting that the allosteric transition has taken place. In accordance with the all-atom simulations, a similar behaviour has been observed analysing

the bottleneck distances in the CG and Elastic Network simulations (data not shown); thus resulting in a coherent representation of the system allosteric movement under the influence of the ligands, for the three difference models. However, such narrow space renders a quantification of the total ionic resistance highly dependent on the multiple bottlenecks and conduction channels along the pathway.

The presence of small fluctuations and the presence of several charged groups along the pathway, renders the purely steric analysis qualitative and even hard to justify. Upon binding of the receptor with the ligands, the allosteric modification alters ionic resistance in several ways: the variations in the electrostatic environment along the pore extension, the fluctuating motion of the protein matrix in the channel, the presence of water that is advected and that alters the hydrophobic content of the pore, the sub-Angstrom modification 
of the pore bottlenecks. These conditions can result in modifications of the barrier crossing rates by orders of magnitude. Once again, the complex interplay of these conditions and their associated timescales rules out a computational approach based on a direct atomistic approach since it would require hundreds of nanoseconds of observation time for monitoring a single ion passage. Consequently, gathering sufficient statistics is out of reach. The proposed scheme instead provides an effective decoupling between the macromolecular motion and the ionic passage, thereby allowing to study the motion of the saline solution during the macromolecular evolution.

The simulation on ionic transport proceeds as follows. MD simulations deliver a time sequence of structural configurations of the neuroreceptor, $\left\{\mathcal{C}_{t}, t=0, t_{\text {sim }}\right\}$. For a prescribed sequence of such configurations, a long-time (hundreds of nanoseconds) LB simulation is performed until the steady-state current, $J_{t} \equiv J_{s s}\left(\mathcal{C}_{t}\right)$, supported by the given configuration at time $t$ is obtained. In passing, we note that this procedure also permits to accumulate significant statistics, due to the the fact that the structural changes of NMDA are pretty slow on the scale of the MD integration. The resulting current $J_{t}$ shows abrupt up and down changes in time, which we associate with opening (closing) of conductive channels within the receptor configuration. Needless to say, the structural dynamics of these channels is extremely rich, with abrupt morphological changes, such as sudden narrowings that eventually quench an otherwise highly conductive channel and vice-versa.

Functional response is quantified by considering both the APO and HOLO versions of NMDA and considering a single 300 ns MD trajectory. Conformational analysis of the receptors and application of the HOLE method exhibit large fluctuations of the pore region by steric analysis, indicating that allosteric transition is effectively taking place (data not shown). Conductance was measured by extracting 100 protein conformations, evenly spaced in time of the last $100 \mathrm{~ns}$ of the all atom simulations of the equilibrated receptor for the putative close and open states.

Figure $7 \mathrm{C}$ shows the conductance (in picoSiemens) as a function of time. From this figure, several bursts of conductance are visible past the open-up event mark the genuine microscopic nature of conductance, driven by the underlying fluctuations of the protein matrix. These fluctuations provide a neat signature of channel opening/closing events but with a clear trend towards stabilisation of the open configuration. It is expected that at longer times, not covered by the present simulations, the open state will likely exhibit a 
consistent amount of short-lived closed configurations.

Summarising, within our procedure, the neuroreceptor is treated as a slow time-changing molecular porous medium, and the geometrical flexibility of LB is leveraged to compute the "electrical permeability" of such molecular porous media "on-the-fly" Succi et al. [47].

\section{Summary}

We have presented a new three-stage multiscale method for the computational study of the NMDA neuroreceptor.

The computational framework combines three distinct representation levels:

i) Homology models for the characterisation of the protein structure, ii) various forms of Molecular Dynamics for the dynamical stability of the NMDA complex and finally iii) lattice Boltzmann simulations of ion transport across the neurotransmitter. The main novelty of our approach relies on LB electrokinetics to analyse the mesoscale flow of ions by taking into account the complete hydrodynamic modes of the saline solution as it flows inside the receptor matrix. It is thus possible to observe directly the effect of electrostatic forces and convection on ionic currents at varying receptor configurational states. To this aim, the usage of mesoscopic representation of the protein via a coarse grained force field (such as OPEP used in this work) with the saline solution described by the Boltzmann picture provides equivalent levels of detail. In more general terms, the three-stage multiscale method described here can be further extended to combine the evolution of the Lagrangian representation of one subsystem, typically simulated via MD, with the eulerian representation of a complementary subsystem, simulated via LB, thereby taking into account the dynamical two-way exchange of forces.

The numerical results on NMDA show clear evidence of allosteric transitions stimulated by binding of Glutamate and Glycine ligands. Ionic transport across the NMDA complex show preliminary agreement with experimental data. Importantly, the provided scheme makes the study of the neuroreceptor functional response viable thanks to the high performances of the MD and LB components. In particular the MD component requires approx. $4 \mathrm{GPU}$ hours per nanosecond, while the LB requires $1 \mathrm{CPU} /$ hour per simulation of electrokinetics, that translates to $0.1 \mathrm{GPU} /$ hour when running on a single GPU.

It is hoped and expected that the present three-stage framework may pave the way to 
the computational study of a variety of multiscale biological processes spanning a broad spectrum of scales in space and time. As presented in the text, such outcome compares very favourably with single-channel patch clamps, thus boding well for future use of the present computational multi-stage methodology to other types of neuroreceptors.

\section{Acknowledgements}

This paper is dedicated to Mike Klein, a trailblazing and highly inspiring figure of molecular simulation at large.

SS wishes to acknowledge funding from the European Research Council under the Horizon 2020 Programme Grant Agreement n. 739964 ("COPMAT"). 


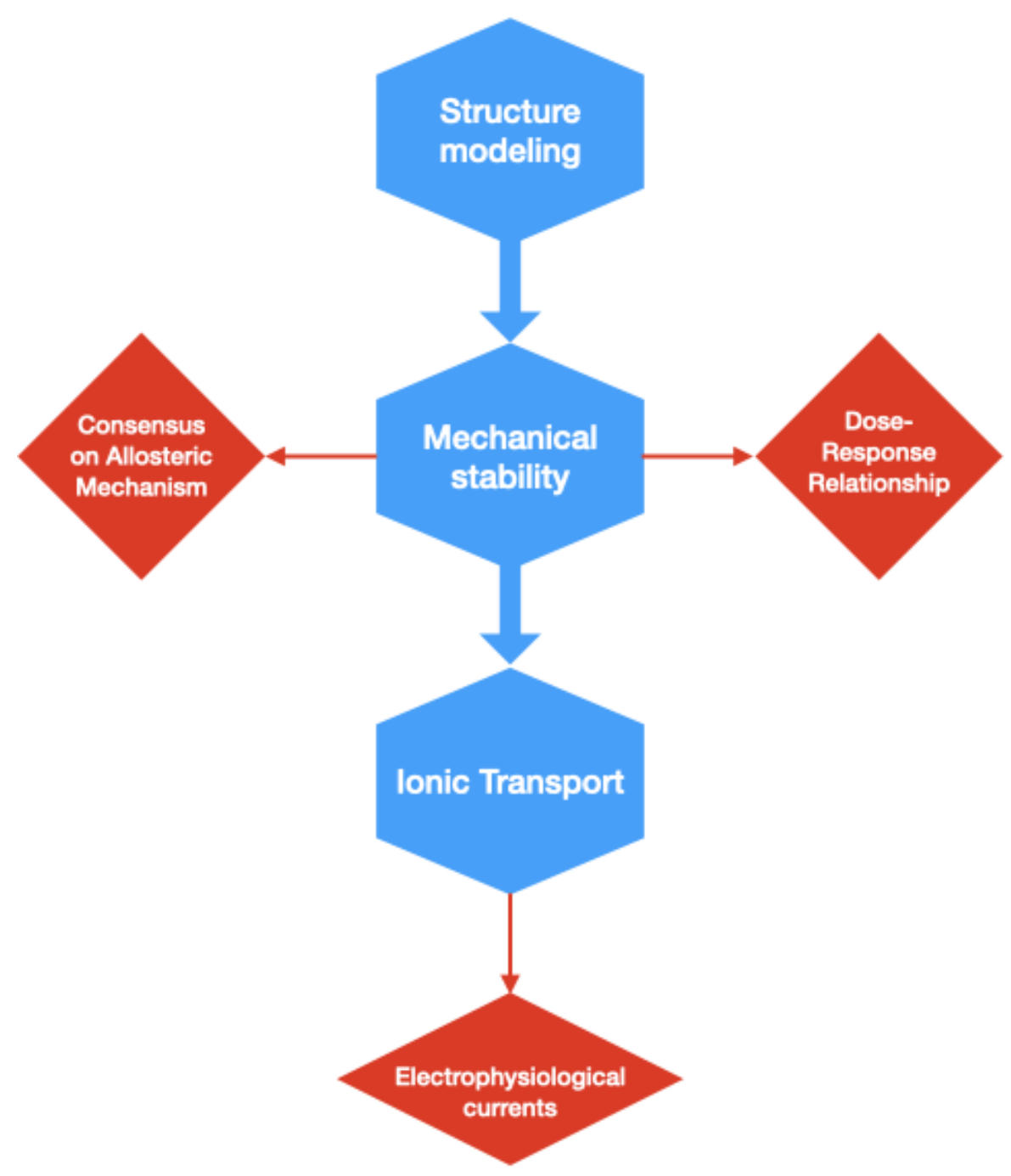

FIG. 1: Protocol Flowchart illustrating the three-level approach to studying the neuroreceptor function. Top: starting from the incomplete crystallographic data, homology and structure modelling allows reconstructing the initial condition. Middle: by applying MD all-atom and coarse grained simulations one can access the allosteric transition and compute affinity to binding, allowing to determine the dose-response relation. Finally, use of the fluctuating protein matrix enables the use of LB to quantify the eletrokinetic content of the receptor, thereby constructing the dose-function relation. 


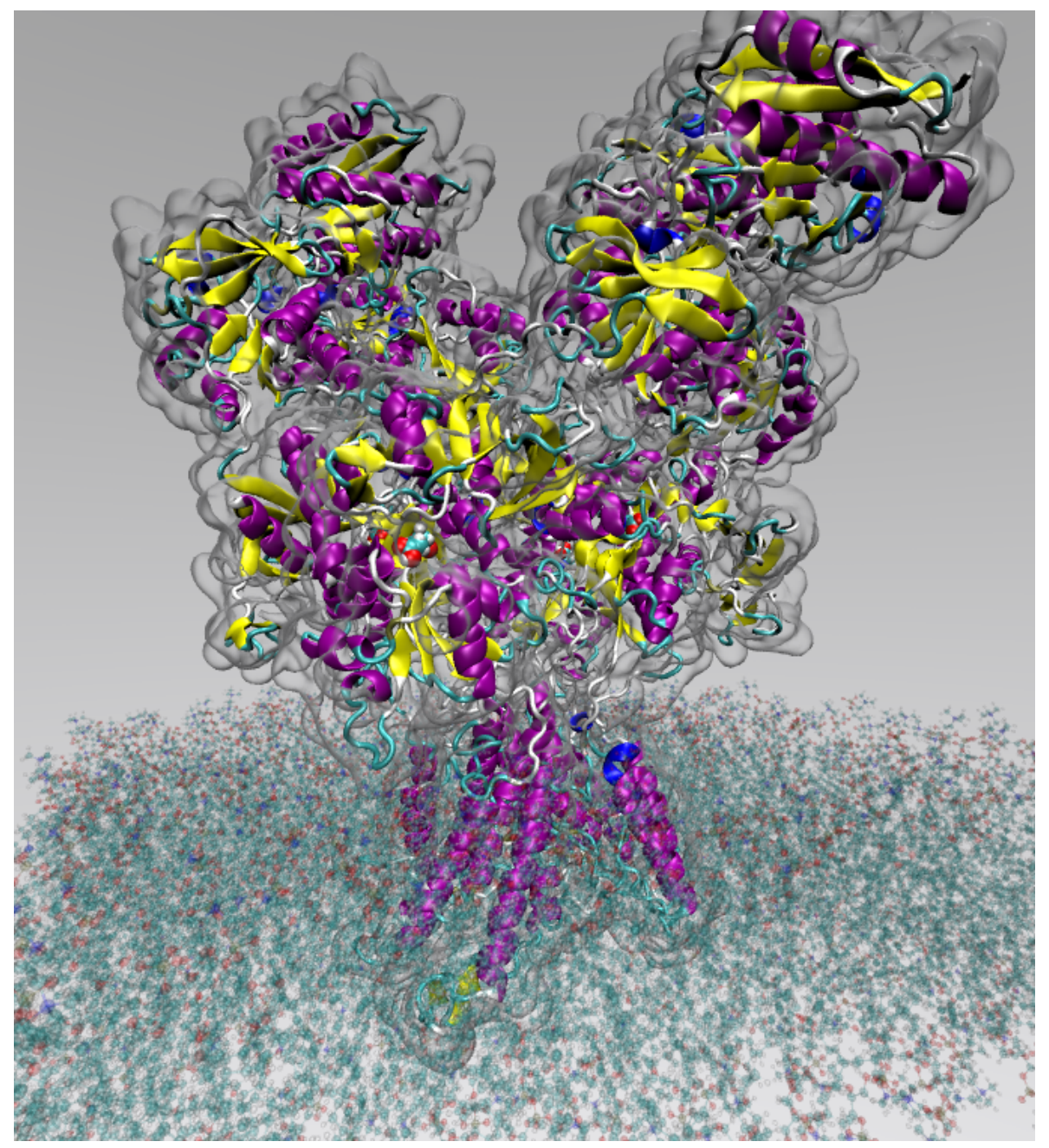

FIG. 2: Reconstructed NMDA receptor embedded in the POPC membrane and with the GLN and GLY ligands. The water solvent has been removed from the visualisation for the sake of clarity. . 


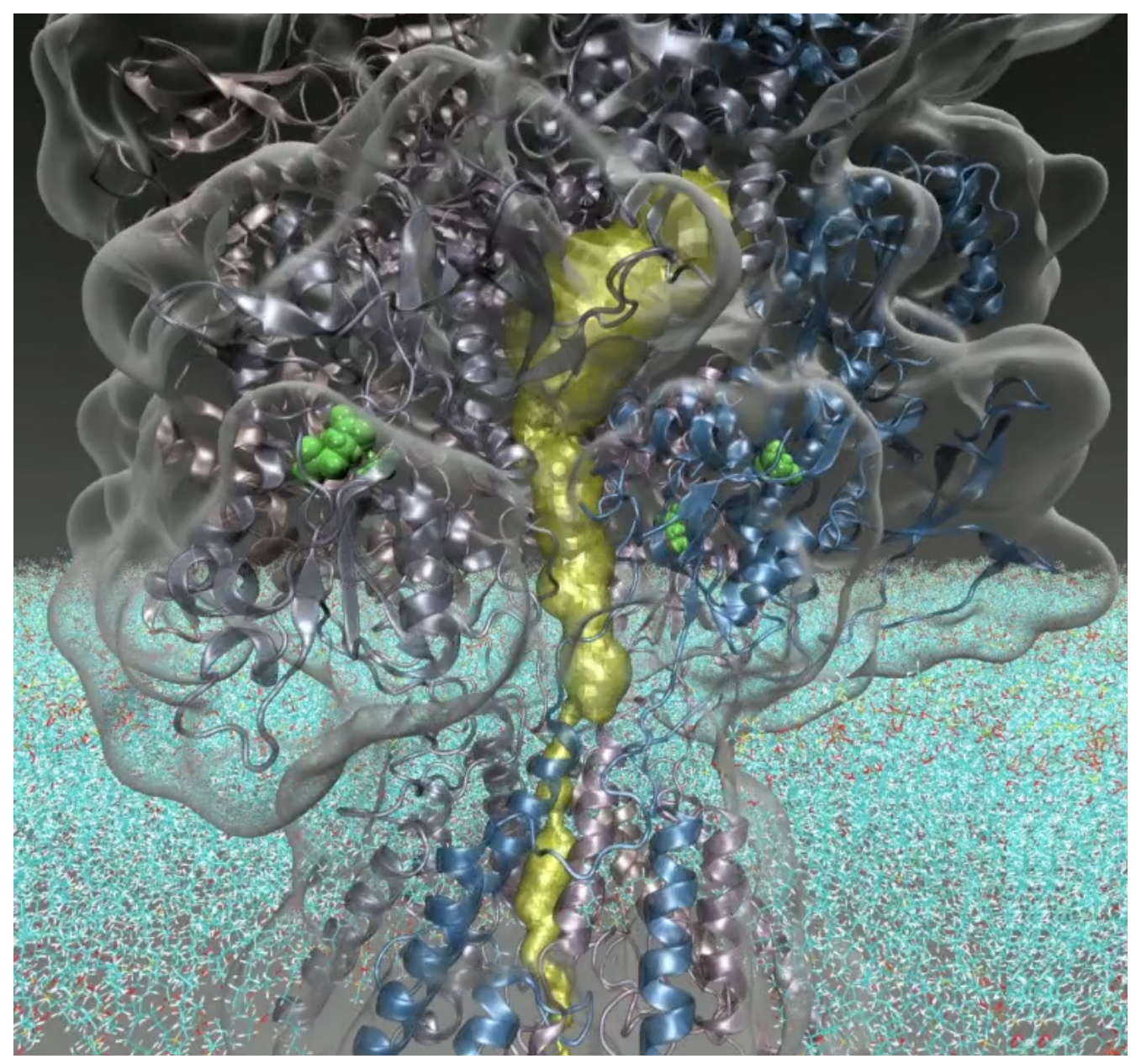

FIG. 3: Example of passage obtained with the HOLE method exemplifying one possible conduction pathway and its narrow steric space . 

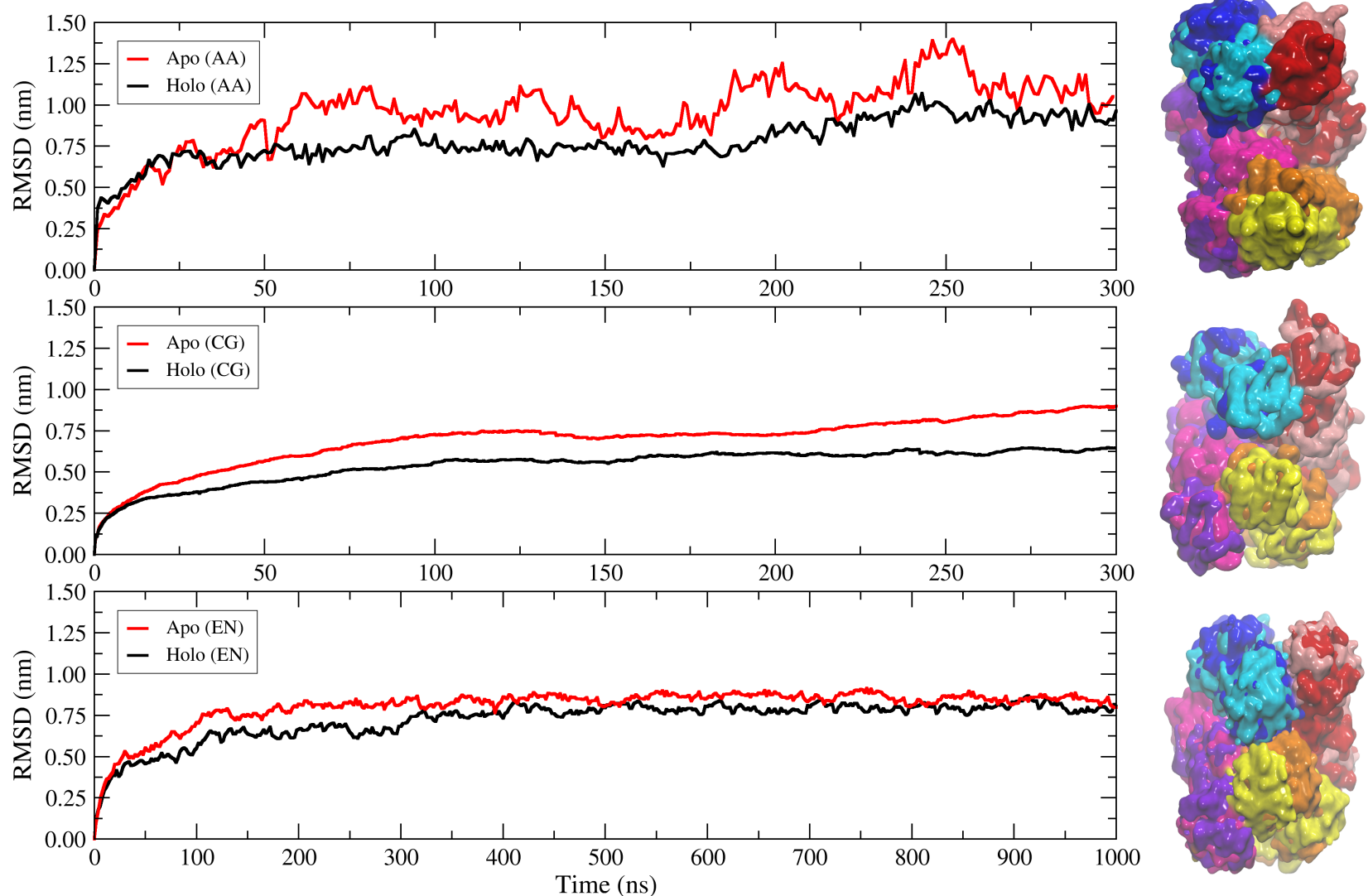

FIG. 4: RMSD of the $\mathrm{C}_{\alpha}$ along the HOLO (black) and APO (red) simulations in the three different levels of detail: all-atom (AA, top), OPEP coarse-grained (CG, middle) and elastic network (EN, bottom). On the right-side of each plot the top views of the superimposed APO and HOLO conformations of the apical lobes (transparent chain-coloured surface representation: APO chains in cyan, pink, yellow and magenta; HOLO chains in blue, red, orange and purple) . 

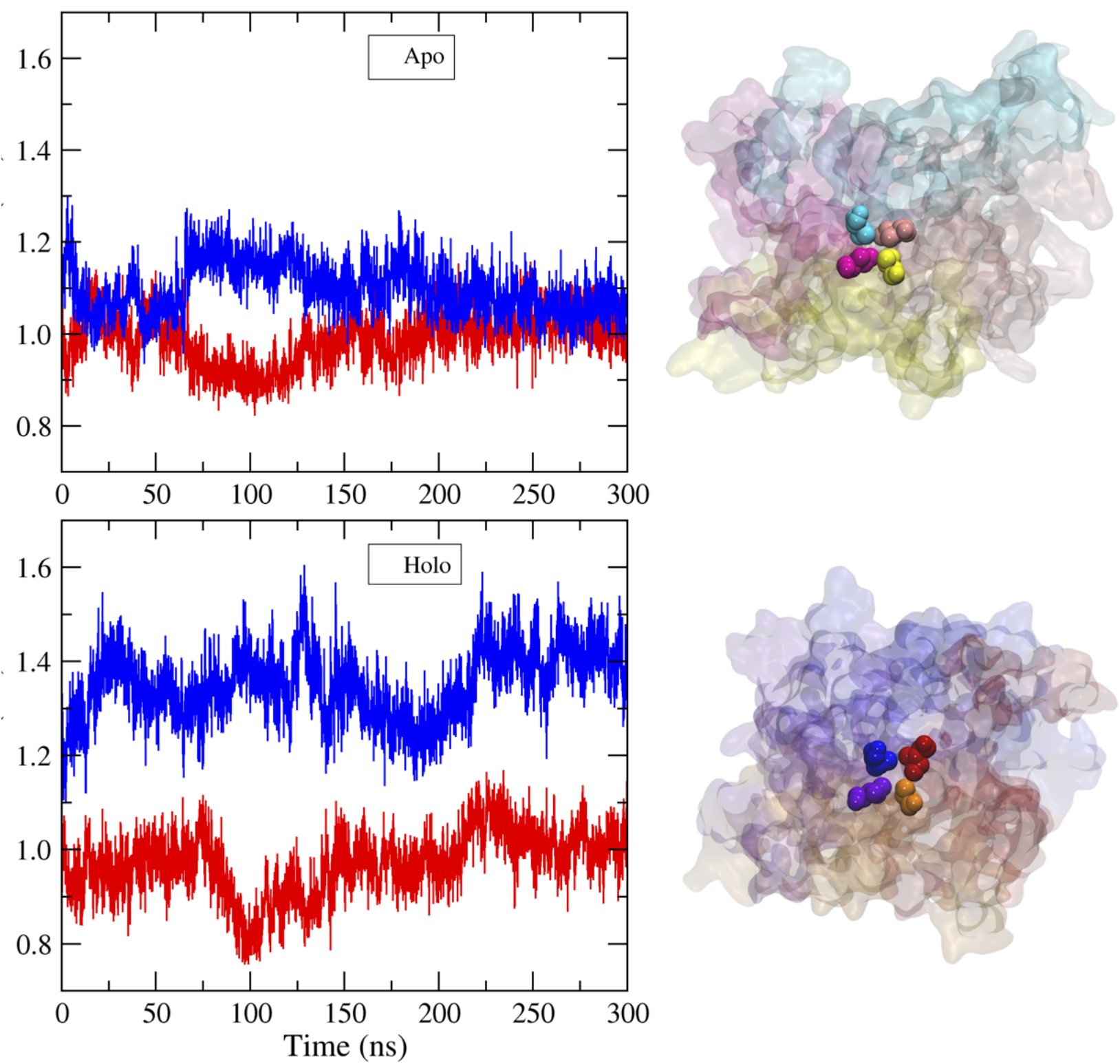

FIG. 5: Time evolution of the two gating bottleneck distances in the NMDA pore for the APO (upper) and HOLO (lower) states. In both panels, in red the distance between the CoM of V632 and V2244 of the two GluN1A monomers, in blue the distance between I1426 and I3039 of the GluN2B monomers. On the right-side of each plot the corresponding APO and HOLO conformations of the bottleneck cross-section (represented as semi-transparent chain-coloured surface) with the four above mentioned residues (APO: V632 cyan, I1426 pink, V2244 yellow and I3039 magenta; HOLO: V632 blue, I1426 red, V2244 orange and I3039 purple). . 


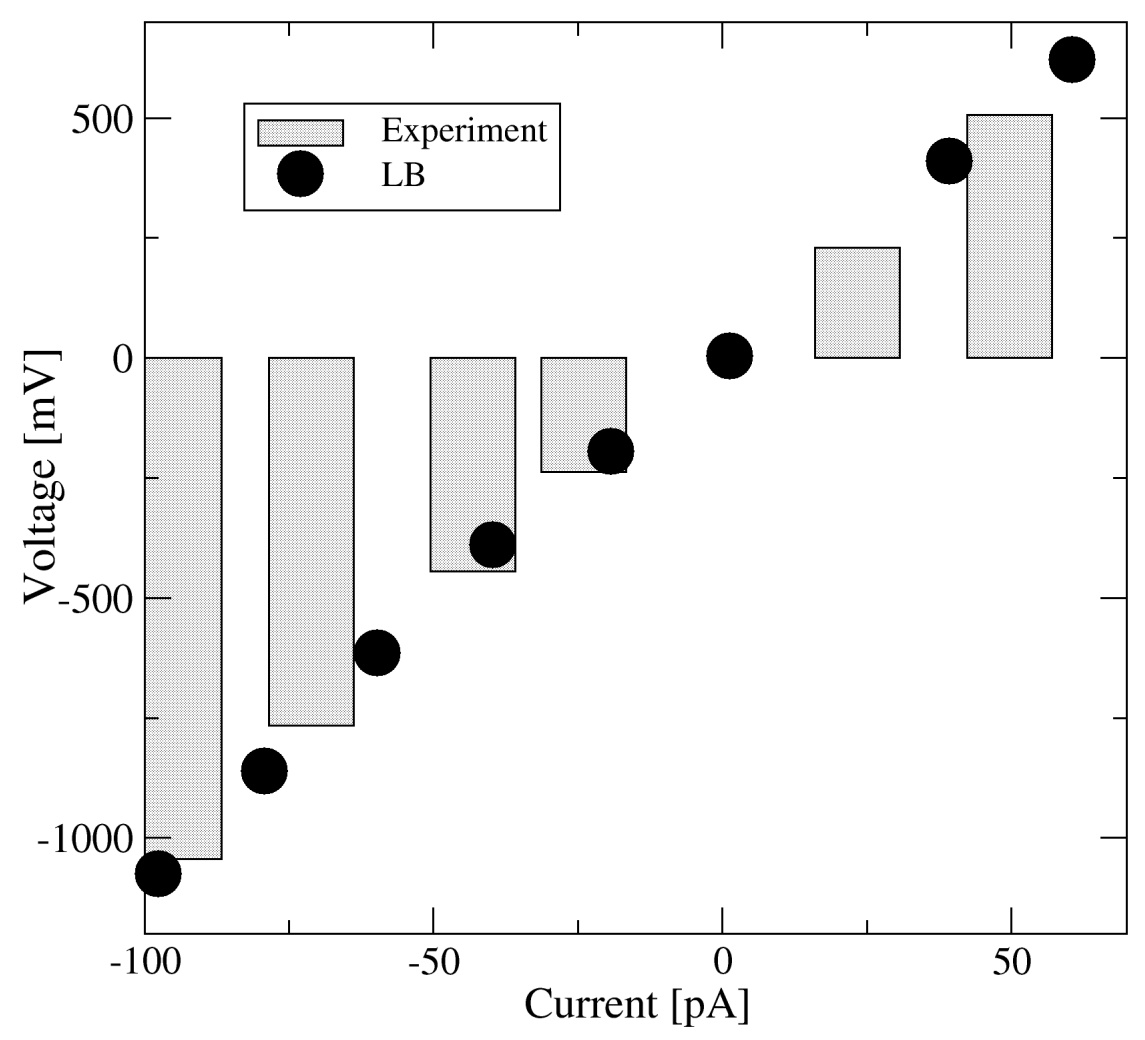

FIG. 6: Characteristic curve of NMDA obtained fromthe LB method and compared to experimental data Cui et al. [38]. 


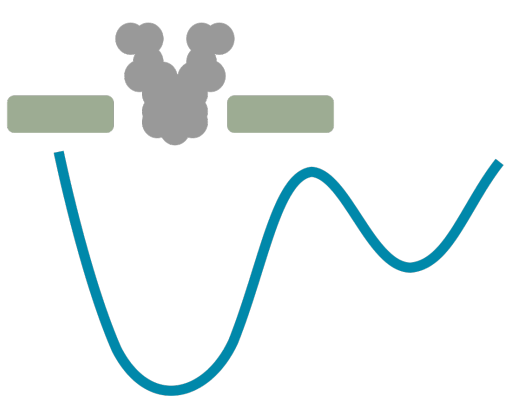

conductance off

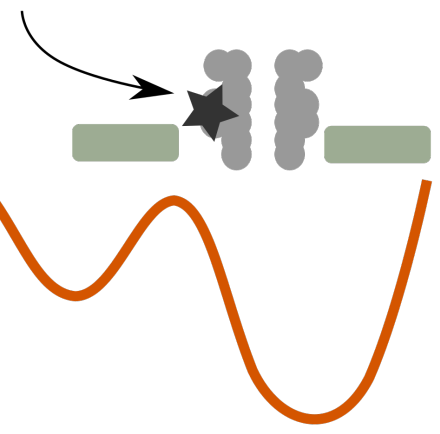

conductance on

\section{A}

B

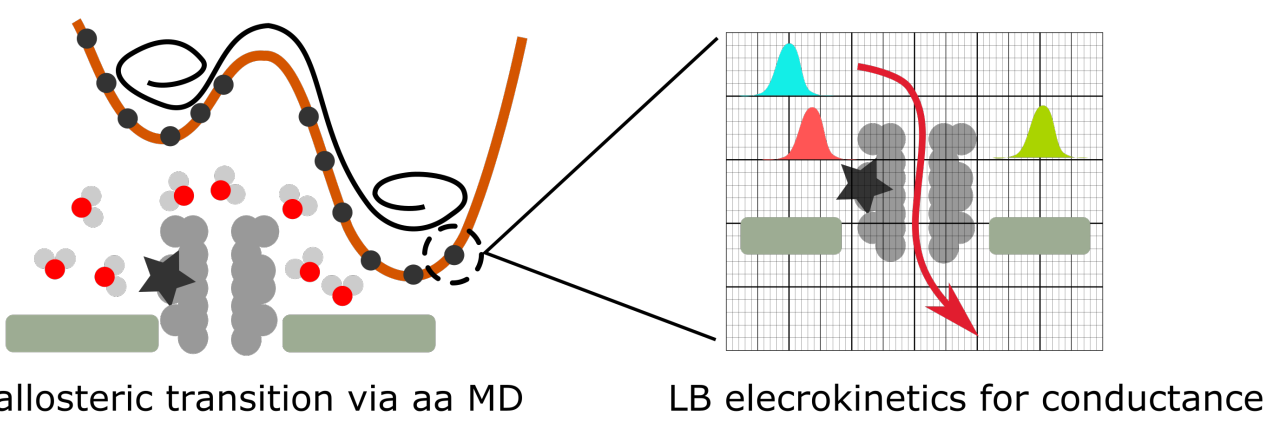

allosteric transition via aa MD

LB elecrokinetics for conductance
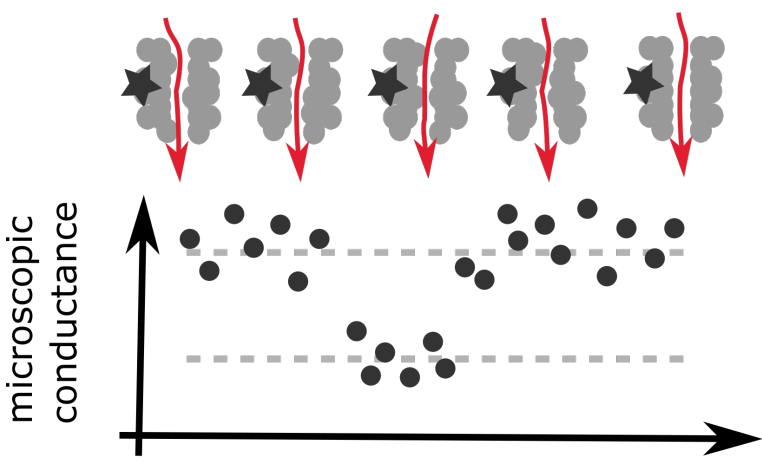

receptor conformations

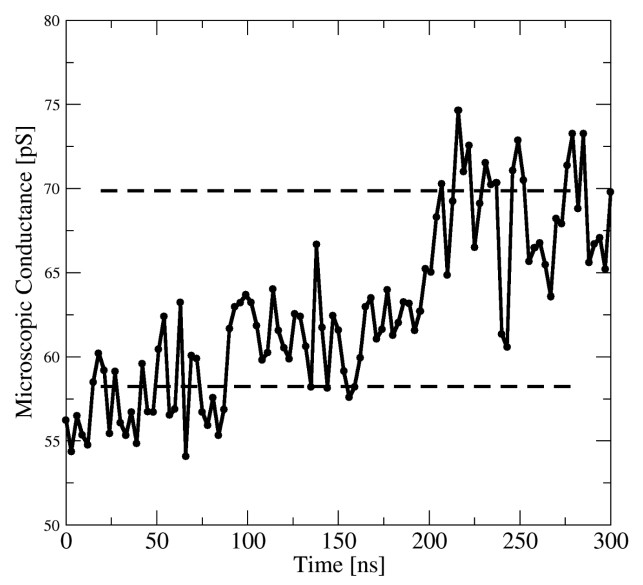

C

FIG. 7: Representation of NMDA evolution upon binding with the ligands and the computational scheme. A: the receptor evolves from the closed to the open state, characterised by a change in conductance from a baseline value to the one associated with the conducting state. $\mathrm{B}$ : MD simulations sample the allosteric evolution of the receptor conformations, characterized by large fluctuations during the $300 \mathrm{~ns}$ single MD trajectory. C: by selecting configurations every 3 ns after binding to ligands, the evolution of conductance is obtained from LB simulations of a saline solution (made of neutral, anionic and cation 24 species), whose data are shown in the rightmost column. The horizontal lines are guides to the eye . 
[1] M. Karplus, M. Levitt, and A. Warshel (2013), www.nobelprize.org/prizes/chemistry/2013.

[2] J.-D. Rochaix, Cell 179, 1012 (2019).

[3] X. Song, M. Ø. Jensen, V. Jogini, R. A. Stein, C.-H. Lee, H. S. Mchaourab, D. E. Shaw, and E. Gouaux, Nature 556, 515 (2018).

[4] M. Trimble, Molecular neuropharmacology, a foundation for clinical neuroscience (McGrawHill, New York, 2002).

[5] E. S. Burnell, M. Irvine, G. Fang, K. Sapkota, D. E. Jane, and D. T. Monaghan, Journal of medicinal chemistry 62, 3 (2018).

[6] J. Dai and H.-X. Zhou, Biophysical journal 104, 2170 (2013).

[7] W. Zheng, H. Wen, G. J. Iacobucci, and G. K. Popescu, Biophysical journal 112, 2589 (2017).

[8] Z. Palmai, K. Houenoussi, S. Cohen-Kaminsky, and L. Tchertanov, PloS one 13, e0201234 (2018).

[9] B. Roux, T. Allen, S. Berneche, and W. Im, Quarterly reviews of biophysics 37, 15 (2004).

[10] M. G. Kurnikova, R. D. Coalson, P. Graf, and A. Nitzan, Biophysical Journal 76, 642 (1999).

[11] O. S. Smart, J. G. Neduvelil, X. Wang, B. Wallace, and M. S. Sansom, Journal of molecular graphics 14, 354 (1996).

[12] N. Eswar, B. Webb, M. A. Marti-Renom, M. Madhusudhan, D. Eramian, M.-y. Shen, U. Pieper, and A. Sali, Current protocols in bioinformatics 15, 5 (2006).

[13] M. J. Abraham, T. Murtola, R. Schulz, S. Páll, J. C. Smith, B. Hess, and E. Lindahl, SoftwareX 1, 19 (2015).

[14] E. Karakas and H. Furukawa, Science 344, 992 (2014).

[15] M.-y. Shen and A. Sali, Protein science 15, 2507 (2006).

[16] S. Jo, T. Kim, V. G. Iyer, and W. Im, Journal of computational chemistry 29, 1859 (2008).

[17] E. L. Wu, X. Cheng, S. Jo, H. Rui, K. C. Song, E. M. Dávila-Contreras, Y. Qi, J. Lee, V. Monje-Galvan, R. M. Venable, et al., Charmm-gui membrane builder toward realistic biological membrane simulations (2014).

[18] M. A. Lomize, A. L. Lomize, I. D. Pogozheva, and H. I. Mosberg, Bioinformatics 22, 623 
(2006).

[19] J. Lee, X. Cheng, J. M. Swails, M. S. Yeom, P. K. Eastman, J. A. Lemkul, S. Wei, J. Buckner, J. C. Jeong, Y. Qi, et al., Journal of chemical theory and computation 12, 405 (2016).

[20] J. Huang and A. D. MacKerell Jr, Journal of computational chemistry 34, 2135 (2013).

[21] Y. Chebaro, S. Pasquali, and P. Derreumaux, J. Phys. Chem. B 116, 8741 (2012).

[22] F. Sterpone, S. Melchionna, P. Tuffery, S. Pasquali, N. Mousseau, T. Cragnolini, Y. Chebaro, J.-F. St-Pierre, M. Kalimeri, A. Barducci, et al., Chem. Soc. Rev. 43, 4871 (2014).

[23] F. Sterpone, P. Nguyen, M. Kalimeri, and P. Derreumaux, J. Chem. Theory. Comput. 9, 4574 (2013).

[24] J. W. Rocks, N. Pashine, I. Bischofberger, C. P. Goodrich, A. J. Liu, and S. R. Nagel, Proceedings of the National Academy of Sciences 114, 2520 (2017).

[25] F. Sterpone, P. Derreumaux, and S. Melchionna, J. Chem. Theory Comput. 11, 1843 (2015).

[26] M. Chiricotto, S. Melchionna, P. Derreumaux, and F. Sterpone, J. Chem. Phys. 145, 035102 (2016).

[27] M. Chiriccotto, S. Melchionna, P. Derreumaux, and F. Sterpone, J. Phys. Chem. Lett. 10, 1594 (2019).

[28] S. Timr, D. Gnutt, S. Ebbinghaus, and F. Sterpone, J. Phys. Chem. Lett. 11, 4206 (2020).

[29] F. Sterpone, S. Melchionna, and P. Derreumaux, J. Phys. Chem. B 122, 1573 (2018).

[30] O. Languin-Cattoen, S. Melchionna, P. Derreumaux, G. Stirnemann, and F. Sterpone, J. Phys. Chem. B 122, 11922 (2018).

[31] M. Fyta, S. Melchionna, and S. Succi, Journal of Polymer Science Part B: Polymer Physics 49, 985 (2011).

[32] S. Succi, The Lattice Boltzmann equation: for fluid dynamics and beyond (Oxford university press, 2018).

[33] U. M. B. Marconi and S. Melchionna, The Journal of chemical physics 134, 064118 (2011).

[34] S. Melchionna and U. M. B. Marconi, EPL (Europhysics Letters) 95, 44002 (2011).

[35] B. Lu, M. J. Holst, J. A. McCammon, and Y. Zhou, Journal of computational physics 229, 6979 (2010).

[36] S. Melchionna and S. Succi, The Journal of chemical physics 120, 4492 (2004).

[37] U. Marini Bettolo Marconi and S. Melchionna, Langmuir 28, 13727 (2012).

[38] C. Cui, M. Xu, and M. Atzori, Molecular pharmacology 70, 1761 (2006). 
[39] S. Raafatnia, O. A. Hickey, M. Sega, and C. Holm, Langmuir 30, 1758 (2014).

[40] U. M. B. Marconi and S. Melchionna, The Journal of chemical physics 135, 044104 (2011).

[41] C. S. Peskin, Acta numerica 11, 479 (2002).

[42] A. Krämer, D. Wilde, K. Küllmer, D. Reith, and H. Foysi, Physical Review E 100, 023302 (2019).

[43] A. Montessori, G. Falcucci, P. Prestininzi, M. La Rocca, and S. Succi, Physical Review E 89, 053317 (2014).

[44] C. J. Williams, J. J. Headd, N. W. Moriarty, M. G. Prisant, L. L. Videau, L. N. Deis, V. Verma, D. A. Keedy, B. J. Hintze, V. B. Chen, et al., Protein Science 27, 293 (2018).

[45] F. Sterpone, S. Doutreligne, T. T. Tran, S. Melchionna, M. Baaden, P. H. Nguyen, and P. Derreumaux, Biochemical and biophysical research communications 498, 296 (2018).

[46] G. Klesse, S. Rao, M. S. Sansom, and S. J. Tucker, Journal of molecular biology 431, 3353 (2019).

[47] S. Succi, E. Foti, and F. Higuera, EPL (Europhysics Letters) 10, 433 (1989). 